\title{
Effect of loading rate on crystallization of metallic glass supercooled liquids
}

\author{
E. Davoodi ${ }^{1}$, M. Hasan ${ }^{1}$, S. Rana ${ }^{2}$ and G. Kumar ${ }^{3 *}$ \\ ${ }^{1}$ Department of Mechanical Engineering, Texas Tech University, Lubbock, TX 79409, USA \\ ${ }^{2}$ Department of Basic Science and Humanities, University of \\ Engineering and Management, Newtown, Kolkata700160, India and \\ ${ }^{3}$ Department of Mechanical Engineering, The University of Texas at Dallas, Richardson, TX 75080, USA
}

\begin{abstract}
Metallic glasses exhibit unique thermoplastic processing capability, which is enabled by their metastable supercooled liquid state below the crystallization temperature. The thermoplastic processing critically depends on the crystallization time (processing time window), temperature (viscosity), applied load, and strain-rate. Among these parameters, the effects of crystallization time and processing temperature have been extensively studied. However, the effects of load and loading rate have not been thoroughly investigated. In this work, we performed a systematic study of load on the supercooled liquid state of three metallic glass formers: Pt-based, Zr-based, and Pd-based. The results show that the load-response of a metallic glass supercooled liquids is strongly composition dependent. The onset temperature of crystallization decreases with increasing load in Pt-based metallic glass whereas for Zr-based and Pd-based metallic glasses the onset temperature remains unchanged. The crystallization peak time is reduced for all three metallic glasses after thermoplastic forming. The results are discussed in terms of nucleation and growth of crystallites in metallic glasses.
\end{abstract}

Keywords: Metallic glass; Thermoplasticforming; Crystallization;

\section{INTRODUCTION}

Metallic glasses (MGs) become pliable above their glass transition temperatures $\left(T_{g}\right)$ because of drastic reduction in viscosity [1-4]. This trait of MGs has been exploited for thermoplastic forming (TPF) operations to make metallic structures at length scales ranging from macro to nano [1-8]. Thermoplastically patterned MG surfaces have been studied to understand the effects of topography on wetting $[5,9]$, reflectance [10], cellular response [11], and catalytic activity [12]. TPF has also been used for sheet making [13], blow molding [14], and extrusion [15]. TPF is performed isothermally above $T_{g}$, where the metallic glass supercooled liquid has limited available time before crystallization $[16,17]$. Therefore, crystallization time is an important parameter because it sets an upper limit for the processing time. Typically, TTT (time-temperature-transformation) diagram is used to determine the crystallization for metallic glass supercooled liquids [1-4]. The use of TTT diagram is based on the assumption that isothermal crystallization time is unaffected by any other parameters except the temperature [18]. However, recent studies have indicated that strain-rates and pressure can influence the crystallization of metallic glasses [19].TPF subjects the metallic glass to varying shear strains (strain-rates) and pressures, which may alter the crystallization times. Therefore, it is important to study the effects of strain-rates and pressure on crystallization of metallic glasses. In this study, we report the results from isothermal crystallization of three metallic glasses after subjecting them to different loading

*corresponding author. E-mail: golden.kumar@utdallas.edu rates and pressures in the supercooled liquid state.

\section{EXPERIMENTAL}

Ingots of Pt-MG $\left(P t_{57.5} C u_{14.7} N i_{5.3} P_{22.5}\right)$ and Pd$\mathrm{MG}\left(\mathrm{Pd}_{43} \mathrm{Cu}_{27} N i_{10} P_{20}\right)$ alloys were prepared by melting the constituents of each alloy in vacuum-sealed quartz tubes. The ingots were melted twice to achieve compositional homogeneity. Subsequently, the alloys were fluxed in $B_{2} O_{3}$ which has been shown to improve the glass forming ability in metallic systems. The fluxed ingots were melted and water-quenched in thinwall quartz tubes. $Z r_{35} T i_{30} C u_{8.25} B e_{26.75}$ (Zr-MG) was purchased from Liquid Metal Technology. The samples were isothermally loaded at different rates from 3.33 to $3333.33 \mathrm{~N} / \mathrm{min}$ for $3 \mathrm{~min}$. The loading experiments were doneusing Instron machine equipped with heating plates. The samples became thin circular discs after pressing. After loading, the samples were quenched in water to retain the structural state. To differentiate the effect of load on crystallization, the samples were isothermallyannealed for the same durations without any load. The crystallization kinetics were characterized using Q20 TA instruments differential scanning calorimetry (DSC).

\section{RESULTS AND DISCUSSION}

To study the influence of temperature on crystallization kinetics, the as-prepared Pt-MG samples were isothermally annealed at three different temperatures as shown in Figure 1. Typically, the onset, the peak, and the shape of crystallization curves are of most interest. The onset and peak times of crystallization decrease with

\section{American Journal of Physical Sciences and Applications US:ISSN 2644-0792}




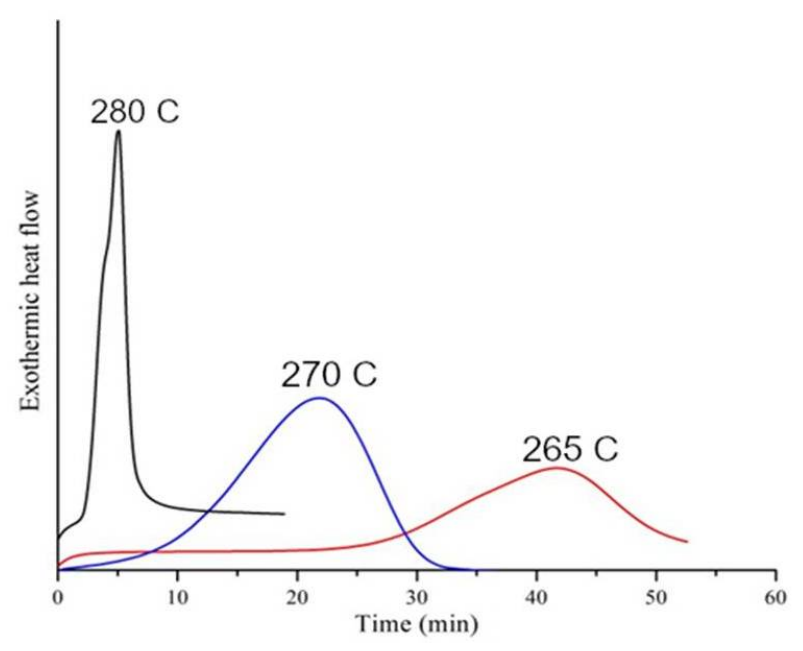

FIG. 1: Isothermal crystallization curves for Pt-MG at three different temperatures.

increasing temperature. The crystallization of MG supercooled liquids is a first order phase transformation, which is controlled by the nucleation and the growth mechanisms. The growth is often the limiting factor as many MGs already contain quenched-in nuclei formed during synthesis. The decrease in crystallization time with increasing temperature is a consequence of higher mobility and growth rate at high temperatures. The crystallization peak also broadens at lower temperature due to nucleation dominant process where the growth takes place over longer times. Similar results were obtained for the other two MGs (Pd-MG and Zr-MG).

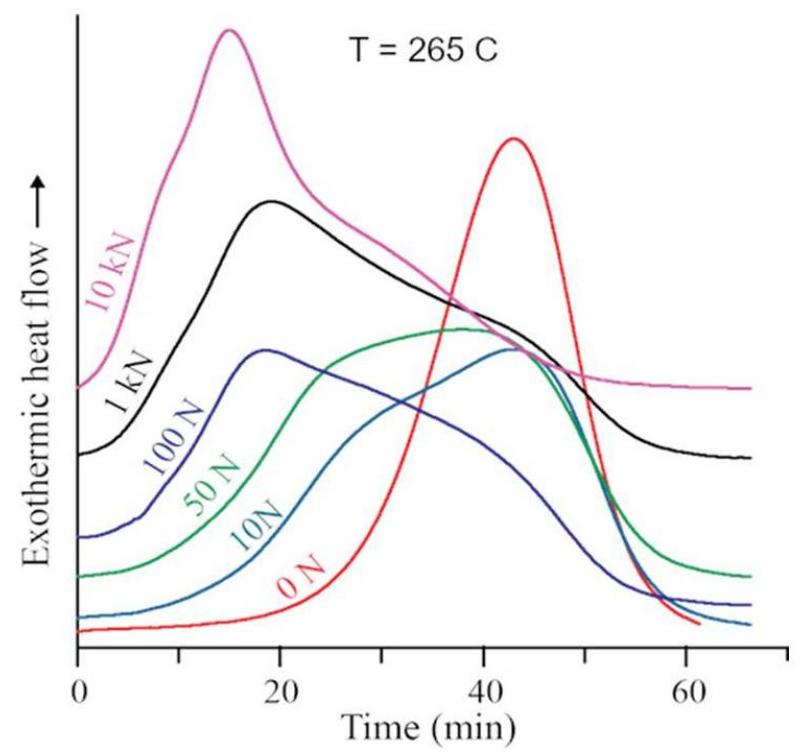

FIG. 2: Isothermal crystallization curves for Pt-MG at $265^{\circ} \mathrm{C}$ after thermoplastic forming at $265^{\circ} \mathrm{C}$ for 3 min under varying loads.

To understand the effect of strain-rate and load, Pt-
MG was subjected to linearly increasing loads for $3 \mathrm{~min}$ at $265^{\circ} \mathrm{C}$. Subsequently, the samples were characterized by isothermal crystallization at $265^{\circ} \mathrm{C}$ using DSC. Figure 2 shows the isothermal DSC curves of Pt-MG samples exposed to different maximum loads and loading rates. For reference, a sample isothermally annealed for 3 min at $265^{\circ} \mathrm{C}$ without any load was also measured (labeled as $0 \mathrm{~N}$ ). The results show that the samples subjected to any non-zero load exhibit two crystallization events. First crystallization peak time continuously decreases with increasing load while the second remains unaffected. Furthermore, the fraction of load-sensitive peak increases with increasing load. Similar results have been reported in previous study [19], and they were explained based on strain-rate assisted crystallization. The MG samples subjected to loads deform radially outward. The outer shells of disc shaped samples experience higher strain and strain-rate than the central parts. Therefore, the two crystallization peaks stem from the central (unaffected) and the outer (strained) parts in loaded samples. The strained fraction increases with increasing load, which can explain the change in magnitudes of two crystallization peaks. It is clear from the isother-

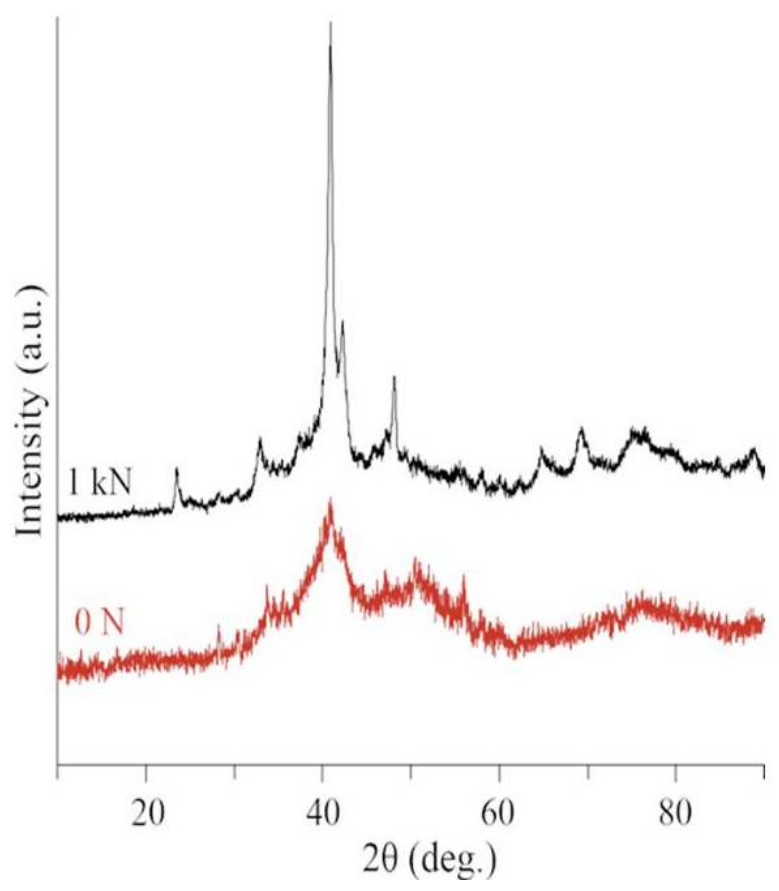

FIG. 3: XRD patterns of Pt-MG exposed to $265^{\circ} \mathrm{C}$ for 3 min without load and under linearly increasing load of $1 \mathrm{kN}$ followed by isothermal annealing at $265^{\circ} \mathrm{C}$ for $50 \mathrm{~min}$ (without load).

mal DSC curves that the structure of MG supercooled liquids changes with strain and strain rate. The deformed material crystallizes faster than the undeformed one. However, crystallization is a combined outcome of nucleation and growth. From the DSC results, it cannot be clearly inferredif straining increases the nucleation or the growth in MG supercooled liquids. To further 
understand the effect of strain, structure of crystallized MGs after straining was studied using X-ray diffraction (XRD). Figure 3 shows the XRD patterns of Pt-MG crystallized after subjecting to load and without load. While it is difficult to identify complexcrystalline phases formed in multi-element MGs, the effect of load on grain structure is evident.The diffraction peaks are sharp for the loaded sample indicating the formation of coarse-grained microstructure. In contrast, broad diffraction peaks indicative of nano-crystalline microstructureare observed for the unloaded sample. These results suggest that thermoplastic deformation facilitates crystal growth by mechanical transport. Higher strain and strain-rate accelerate crystallization of MG supercooled liquids by assisting in growth process. The amount of load (within tested range) does not directly influence the crystallization but affects the strain and strain-rate and hence the crystallization.

Isothermal loading experiments followed by DSC were also performed on $\mathrm{Zr}-\mathrm{MG}$ and $\mathrm{Pd}-\mathrm{MG}$ samples. Figure 4 shows the DSC curves for these two MGs after subjecting them to different loads in the supercooled liquid state. The results for Pd-MG and $\mathrm{Zr}-\mathrm{MG}$ are different than that of Pt-MG. There is no clear onset of crystallization peak (incubation time) in isothermal DSC curves of Pd-MG and Zr-MG even when the temperatures were significantly lower than the constant heating rate crystallization temperatures. The incubation time for crystallization is typically associated with nucleation. The absence of incubation time indicates that Pd-MG and Zr-MG already contain nuclei formed during synthesis or thermoplastic forming. These pre-existing nuclei start growing spontaneously during isothermal DSC measurements. Surprisingly, the Pd-MG and Zr-MG have large glass forming ability, critical sample sizes, and supercooled liquid temperature range. Despite these superior glass forming metrics, the thermal stability of $\mathrm{Pd}-\mathrm{MG}$ and $\mathrm{Zr}-\mathrm{MG}$ supercooled liquids is inferior to Pt-MG. The effect of loading rate is similar on all three MGs. The crystallization peaks shift to shorter times but two-peak behavior is observed only in Pt-MG and with lesser extent in Pd-MG.

\section{CONCLUSIONS}

Isothermal DSC analysis was employed to characterize the effect of plastic deformation on metallic glass supercooled liquids. The results show that loading effects are alloy sensitive but crystallization is accelerated by loading in all three metallic glasses studied in this work. The structural characterization reveals that loading predominantly affects the growth of crystals.

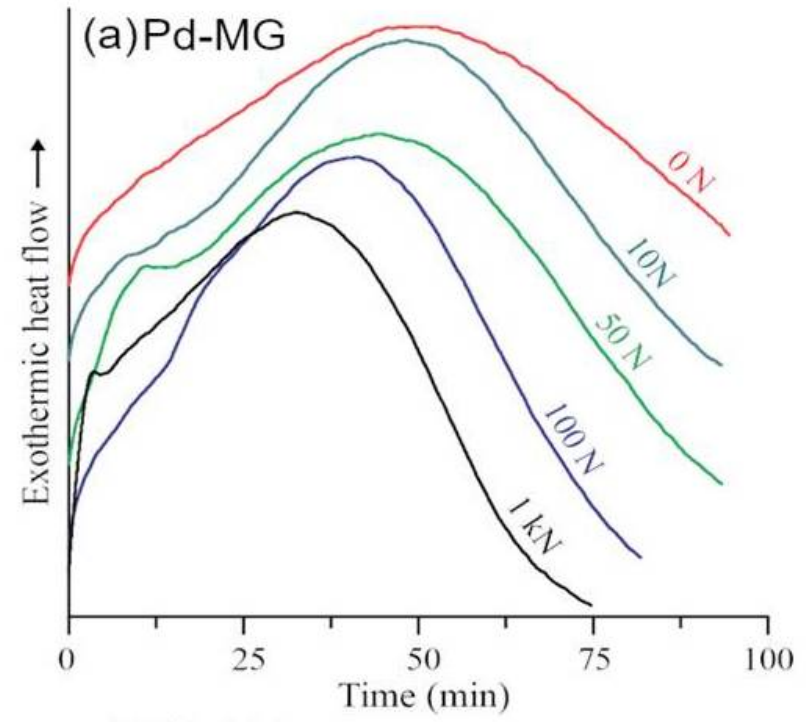

(b) Zr-MG

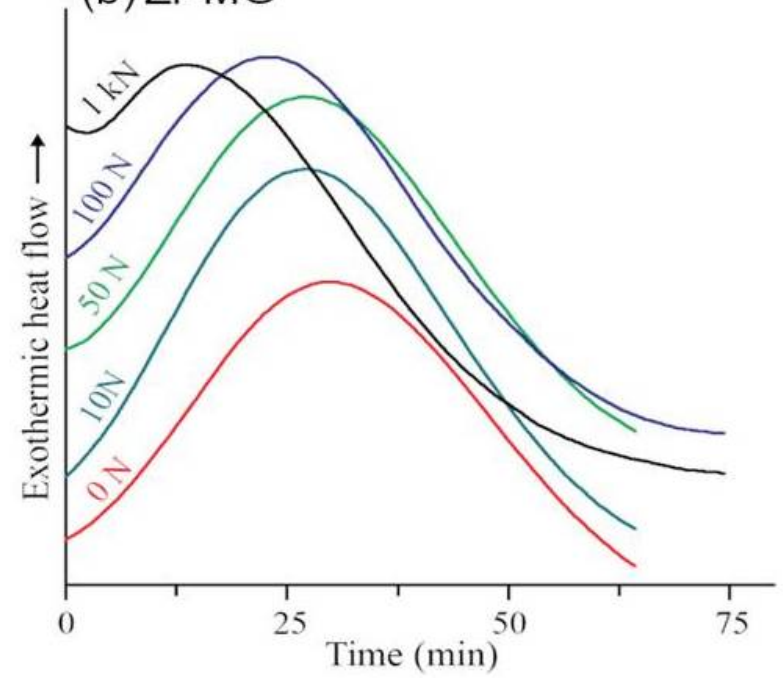

FIG. 4: Isothermal crystallization curves for Pd-MG (a) and Zr-MG (b) conducted at $355^{\circ} \mathrm{C}$ and $405^{\circ} \mathrm{C}$, respectively after thermoplastic forming at the same temperatures under different loads (for $3 \mathrm{~min}$ ).

\section{ACKNOWLEDGEMENTS}

The work was supported by National Science Foundation through awards CMMI-1921435 and CMMI1919445 .
[1] J.Schroers, JOM, 57, 35 (2005).

[2] G. Kumar, H.X. Tang, and J. Schroers, Nature 457, 868 (2009).
[3] J. Schroers ,Adv. Mater, 22, 1566 (2010).

[4] G. Kumar, A. Desai and J. Schroers, Adv. Mater., 23, 461 (2011). 
[5] M. Hasan, J. Schroers, and G. Kumar, Nano Lett. 15, $963(2015)$.

[6] L. Liu, M. Hasan, and G. Kumar, Nanoscale 6, 2027 (2014).

[7] M. Hasan and G. Kumar, Scripta Mater, 123, 140 (2016).

[8] M. Hasan and G. Kumar, Nanoscale, 9, 3261 (2017).

[9] HS Arora, Q. Xu, Z. Xia, YH Ho, NB Dahotre, J. Schroers, and S. Mukherjee, Scripta Mater. 69, 732 (2013).

[10] HJ Tarigan, N. Kahler, NS Ramos, G. Kumar, A A Bernussi, Appl. Phys. Lett., 107, 21903 (2015).

[11] J. Schroers, G. Kumar, TM Hodges, S. Chan, and TR Kyriakised, JOM, 61, 21 (2009).

[12] M. Carmo, RC Sekol, S. Ding, G. Kumar, J. Schroers, and AD Taylor, ACS Nano, 5, 2979 (2011).

[13] R. Martinez, G. Kumar, and J. Schroers, Scr. Mater. 59,
187 (2008).

[14] J. Schroers, T. M. Hodges, G. Kumar, H. Raman, A. J. Barnes, and T. A. WaniukMater. Today 14, 14 (2011).

[15] HM Chiu, G. Kumar, J. Blawzdziewiczand J. Schroers, Scr. Mater. 61, 28 (2009).

[16] Z. Hu, C. S. Meduri, J. Blawzdziewicz, and G. Kumar, Nanotechnology30, 075302 (2018).

[17] G. Kumar, J. Blawzdziewicz, and J. Schroers, Nanotechnology 24, (2013).

[18] E. B. Pitt, G. Kumar and J. Schroers,J. Appl. Phys., 110, 43518 (2011).

[19] 19. Z. Shao, JP Singer, Y. Liu, Z. Liu, M. Gopinadhan, CS O'Hern, J. Schroers, C. Osuji, Phys. Rev. B, 91, 20301 (2015). 